allow youth and elders to experience grandparent-like relationships when grandparents or grandchildren may not be available to each other, giving consideration to contemporary demographic, structural, and historical influences affecting availability of familial intergenerational relationships, theories addressing the imperative of intergenerational relationships, and evidence of the impact of non-familial intergenerational contact on youth and on elders.

\section{RESILIENCES, RESOURCEFULNESS, AND GRANDPARENTING}

C. Musil, M. ${ }^{1}$, J.A. Zauszniewski, Ph.D. ${ }^{2}$,

S.E. Givens, Ph.D. ${ }^{2}$, C. Henrich, $\mathrm{MSN}^{2}$,

M. Wallace, BSN ${ }^{2}$, A. Jeanblanc, $\mathrm{MA}^{2}$,

C.J. Burant, Ph.D. ${ }^{2}$, 1. Case Western Reserve University,

Cleveland, Ohio, United States, 2. Case Western Reserve

University, Cleveland, $\mathrm{OH}, \mathrm{USA}$

Little research using resilience models or measures has been conducted with older adults, and specifically grandparents, although there has been research on resilience focused on children and families. Current theoretical and empirical work examining resilience focuses on the interplay between broad social determinants of health, family and neighborhood factors, and individual factors (physiologic, psychologic, genetic and neurochemical influences on brainbehavior connections) that affect resilience. This presentation describes the major conceptualizations of resilience; factors from "society to cells" that influence resilience capacity and behavior; resourcefulness, an indicator of resilience, comprised of cognitive and behavioral strategies to manage adversity, reduce stress, and promote health; interventions to increase resilience and resourcefulness; and the application of this knowledge to grandparents, grandparenting and grandparent caregiving to grandchildren. The current state of the science and future directions for research and practice will be discussed.

\section{GRANDPARENTING AND HEALTH}

\section{J. Yorgason, B. ${ }^{1}$, M. Serraro Hill' ${ }^{2}$, 1. Brigham Young} University, Provo, Utah, United States, 2. Brigham Young University, Provo, UT, USA

The health of grandparents can have a significant and lasting impact on relationships with grandchildren. In this presentation, we will review grandparent/grandchild relationships when grandparents are healthy, when they contract chronic illness and need care, and when they are in the process of dying. When healthy, grandparents are often more involved physically in their grandchildren's lives, participating in various leisure activities together. However, when affected by chronic illness, interactions with grandchildren may become limited, or changed. Changes in the relationship may be especially unique when dementia or caregiving are involved, as both generations experience some role reversal. Further, when grandparents contract terminal illnesses or are in the process of dying, both generations may experience a greater closeness. Even after death, grandchildren carry a legacy from their grandparents in their hearts. The grandparent/grandchild relationship often experiences both positives and negatives that are blended together and balanced across time.

\section{SESSION 1460 (SYMPOSIUM)}

\section{NEW DEVELOPMENTS IN COGNITIVE AGING RESEARCH}

Chair: G. Hueluer, University of Zurich, Zurich, Zurich

Discussant: H. Dodge, Oregon Health \& Science University and University of Michigan, PORTLAND, Oregon

Due to socio-demographic changes toward an aging population, cognitive aging research is gaining societal and practical importance. Current research is focused on describing age-related and mortality-related trends in cognitive performance, studying potential causes of these changes, identifying factors that promote maintenance of cognitive functioning, and developing interventions based on these research findings. The goal of this research group is to showcase new developments in cognitive aging research dealing with cognitive changes with age and impending death as well as with effects of cognitive training. Hülür et al. examine heterogeneity in trajectories of cognitive change in the last months of life based on routine assessments from 30,000+ Swiss nursing home residents. Robitaille et al. use data from the Swedish OCTO-Twin and the UK Newcastle 85+ studies to examine heterogeneity in terminal decline of global cognition. Thorvaldsson examines pros and cons of terminal decline as a generic model of cognitive aging and associations with cardiovascular health. Bielak et al. use data from the Synapse randomized control trial and evaluate whether intraindividual variability in cognitive speed improves as a result of the cognitive intervention. The discussion by Hiroko Dodge will focus on how these approaches contribute to our understanding of processes of cognitive aging and how they can be utilized to promote maintenance of cognitive functioning in old age.

\section{COGNITIVE CHANGE AT THE END OF LIFE IN NURSING HOME RESIDENTS: DIFFERENTIAL TRAJECTORIES OF TERMINAL DECLINE}

G. Hueluer ${ }^{1}, \mathrm{H}$. Wolf, $\mathrm{PhD}^{2}$, F. Riese, $\mathrm{PhD}^{3}$, N. Theill, $\mathrm{PhD}^{4}$, 1. University of Zurich, Zurich, Zurich, Switzerland, 2. Psychiatric Services Graubuenden, St. Moritz, Switzerland, 3. Psychiatric University Hospital Zurich, Division of Old Age Psychiatry, Zurich, Switzerland; University Research Priority Program "Dynamics of Healthy Aging”, University of Zurich, Switzerland, 4. University Research Priority Program "Dynamics of Healthy Aging”, University of Zurich, Switzerland

Research has widely documented that cognitive performance steeply declines with nearing death. It is unclear whether these changes are normative, based on pathologies associated with (preclinical) dementia, or both. We analyzed heterogeneity in trajectories of terminal cognitive change in Swiss nursing home residents by performing a longitudinal latent class analysis based on routine assessments with the Cognitive Performance Scale (CPS). We used 143,052 observations from 30,054 residents $(69 \%$ women, average age at death $=87$ ) in the last three years of life. We identified three distinct classes based on longitudinal trajectories of the CPS. Residents with severe impairment $(30 \%)$ showed less terminal change compared with other groups. Furthermore, class membership was related to functional health and 
depressive symptoms. Our findings suggest the majority of residents experience terminal change, with the exception of those at already high levels of impairment. Furthermore, late-life cognitive change is related to functional and mental health.

\section{IS COGNITIVE DECLINE BEFORE DEATH IN THE OLDEST OLD A UNIVERSAL PHENOMENON?}

A. Robitaille ${ }^{1}$, D. Cadar, $\mathrm{PhD}^{2}$, A. Koval, $\mathrm{PhD}^{3}$, C. Jagger, $\mathrm{PhD}^{4}$, B. Johansson, $\mathrm{PhD}^{5}$, S. Hofer, $\mathrm{PhD}^{6}$, A. Piccinin, $\mathrm{PhD}^{7}$, G. Muniz-Terrera, $\mathrm{PhD}^{8}$, 1. Department of Psychology, Université du Québec à Montréal, Montreal, Quebec, Canada, 2. Department of Epidemiology and Public Health, University College London, London, UK, 3. Department of Psychology, University of Victoria, Victoria, BC, Canada, 4. Institute of Health and Society, Newcastle University, Newcastle upon Tyne, UK, 5. Department of Psychology, University of Gothenburg, Gothenburg, Sweden, 6. Department of Psychology, University of Victoria, Victoria, BC, Canada, 7. Department of Psychology, University of Victoria, Victoria, BC, Canada, 8. Centre for Dementia Prevention, University of Edinburgh, Edinburgh, UK

We investigated the heterogeneity in end of life cognitive decline in two European longitudinal studies of the oldest old: the OCTO-Twin and the Newcastle 85+ Study. Using a coordinated analytical approach, we identified unobserved groups of individuals with similar trajectories of cognitive decline at the end of life by fitting Tobit Growth Mixture Models to Mini-Mental State Examination scores. In both studies, the current analyses consistently identified two groups of individuals whose cognitive decline at the end of life were distinct: one group did not exhibit an ostensible rate of decline, another group experienced steep decline in measures of global cognition within each study. In OCTO-Twin, accelerated decline was found in only one group. Our results showed heterogeneity in cognitive decline at the end of life in the oldest old across two different European countries and suggest that terminal decline is not necessarily a normative process.

\section{TERMINAL DECLINE AS A GENERIC MODEL OF COGNITIVE AGING}

V. Thorvaldsson, University of Gothenburg, Gothenburg, Vastra Gotaland, Sweden

Cognitive terminal decline (TD) refers to acceleration in an individual decline trajectory with an onset at some specific time (months, years) prior to death. Previous studies provide strong evidence of a large inter-individual differences in the onset of TD, in which some show an acceleration many years prior to death while others are never affected. In the present analysis we further evaluate the pros and cons of TD as a generic model of cognitive aging with the specific purpose to capture inter-individual difference in change trajectories. More specifically we provide examples of the potential role of cardio-vascular health-related variables derived from a representative population-based sample (the $\mathrm{H} 70$ study). The findings show superiority of the time-to-death time structure, in comparison to age-based or time-in-study, to account for inter-individual difference in the change trajectories, and that compromised vascular health in general is associated with earlier onset of TD.
REDUCING INTRAINDIVIDUAL VARIABILITY IN COGNITIVE SPEED VIA PRODUCTIVE ACTIVITY ENGAGEMENT: THE SYNAPSE PROJECT

A.A. M. Bielak ${ }^{1}$, C. Brydges, $\mathrm{PhD}^{2}$, D.C. Park, $\mathrm{PhD}^{3}, 1$. Colorado State University, Fort Collins, Colorado, United States, 2. Colorado State University, Fort Collins, CO, USA, 3. The University of Texas at Dallas, Dallas, Texas, USA

Intraindividual variability (IIV) in cognitive speed has the potential to be a sensitive outcome measure for evaluating cognitive improvement from lifestyle interventions. Using the Synapse Project $(\mathrm{n}=181)$, a randomized controlled trial to improve cognitive ability, we evaluated if older adults who participated in productive engagement (i.e., active learning of quilting, digital photography, or both) showed a reduction in IIV compared to those in receptive engagement (i.e., using existing knowledge via social outings or rote cognitive tasks.). All participants completed their condition for 14 weeks. IIV was based on three versions of a RT flanker task. Complier average casual effect modeling was used with compliance set at 210 hours. The models indicated that compliers in the productive engagement groups did not show any significant change in their IIV. The results demonstrate that even an intensive activity intervention may not be sufficient to cause significant improvement in IIV.

\section{SESSION 1465 (SYMPOSIUM)}

\section{OLDER TRAUMA-EXPOSED MALE AND FEMALE VIETNAM VETERANS: CLINICAL ISSUES AND INNOVATIONS}

Chair: P. Bamonti, VA Boston Healthcare System, Roslindale, Massachusetts

Co-Chair: K. O’Malley, VA Boston Healthcare System, Boston, Massachusetts

Discussant: E.H. Davison, VA Boston Healthcare System, VA National Center for PTSD, Boston University School of Medicine, Boston, Massachusetts

As Vietnam Veterans age into older adulthood, the consequences of lifetime trauma exposure can manifest, including mental and physical health morbidities. Identification of risk and protective factors is needed for the development and tailoring of interventions to mitigate the impact of trauma on wellbeing. This symposium focuses on clinical issues and innovations relevant to research and clinical work with Vietnam Veterans. We begin with research examining risk factors and correlates of mental and physical health outcomes in Vietnam Veterans. First, we will present research examining risk associations linking traumatic stress exposures and mental health correlates with current health-related outcomes in Vietnam era women veterans. Next, we will present findings examining cognitive and other psychological factors related to positive and/or negative outcomes associated with past combat exposure in Vietnam era combat veterans. The program will then shift to innovative interventions for late life PTSD and stress reactions. Pre- and post-group data on veterans who participated in a Later-Adulthood Trauma Reengagement (LATR) group will be presented. The presenter will discuss the application of the LATR model in the treatment of late life PTSD symptomatology, and present program improvement recommendations based on the findings. Fourth, we 